\title{
EVALUACIÓN PRELIMINAR DEL EFECTO DEL QUITOSANO Y CASCARA DE NARANJA EN LA COAGULACIÓN-FLOCULACIÓN DE AGUAS RESIDUALES
}

\section{PRELIMINARY EVALUATION OF THE EFFECT OF CHITOSAN AND ORANGE PEEL IN THE COAGULATION-FLOCCULATION OF WASTEWATER}

\author{
Yesenia Campo Vera ${ }^{1}$, Mónica Alexandra Delgado ${ }^{2}$, Yuly Roa ${ }^{3}$, Gustavo Mora ${ }^{3}$, Javier Carreño Ortiz ${ }^{4}$
}

\begin{abstract}
${ }^{1}$ Magister en Ciencia y tecnología de los alimentos, Directora de investigación. Instituto Superior de Educación Rural, Colombia, e-mail: yesenia.campo.vera@gmail.com, (Dhttps://orcid.org/0000-0003-4658-8113; ${ }^{2}$ Magister en Salud Ocupacional y Ambiental, Docente tiempo completo. Centro de Gestión Agroindustrial de Arauca, Servicio Nacional de Aprendizaje, SENA. Colombia, e-mail: monykd@gmail.com; ${ }^{3}$ Técnico en Manejo Ambiental y Saneamiento básico, Aprendiz. Centro de Gestión Agroindustrial de Arauca, Servicio Nacional de Aprendizaje, SENA. Colombia, e-mail: yulyroa@hotmail.es; ${ }^{4}$ Magister en Innovación, Docente planta. Centro de Gestión Agroindustrial de Arauca, Servicio Nacional de Aprendizaje, SENA. Colombia, e-mail: jcarrenoo@sena.edu.co
\end{abstract}

Rev. U.D.C.A Act. \& Div. Cient. 21(1):565-572, Julio-Diciembre 2018 https://doi.org/10.31910/rudca.v21.n2.2018.990

Artículo de acceso abierto publicado por Revista U.D.C.A Actualidad \& Divulgación Científica bajo una licencia Creative Commons CC BY-NC 4.0

\section{RESUMEN}

Las aguas de desecho dispuestas en una corriente superficial, lagos, ríos, mar, sin ningún tratamiento, ocasionan graves inconvenientes de contaminación, que afectan la flora y la fauna. Estas aguas residuales, antes de ser vertidas en las masas receptoras, deben recibir un tratamiento adecuado, capaz de modificar sus condiciones físicas, químicas y microbiológicas, para evitar que su disposición cause la alteración y la degradación de los ecosistemas asociados y problemas de salud pública. La investigación tuvo como objetivo evaluar el efecto de mezclas de quitosano y extracto acuoso de la cáscara de naranja, a diferentes concentraciones, en el proceso de coagulación-floculación, de aguas residuales, para lo cual, se realizó una prueba de jarras con agitación rápida y lenta, para evaluar turbidez (NTU), demanda química de oxígeno (DQO), demanda bioquímica de oxígeno (DBO), sólidos suspendidos totales (SST) y SS (sólidos sedimentables). Los resultados mostraron que todos los tratamientos presentaron diferencias significativas $(\mathrm{p}<0,05)$, con la muestra control y fueron eficientes en la reducción de turbidez, DBO, DQO, SST, SS. El tratamiento que combinó quitosano y extracto acuoso de cáscara de naranja (50-50\%), a un $\mathrm{pH}$ de 5,5, disminuyó significativamente $(\mathrm{p}<0,05)$ la turbidez, en $79 \%$, demostrando, de manera preliminar, que el extracto acuoso de la cáscara de naranja acidifica la mezcla e incre- menta la formación de flóculos aglomerados en una fase liviana de las muestras, convirtiéndose en un agente eficiente, para ser usado en el tratamiento de aguas residuales.

Palabras clave: carga orgánica, coagulantes, contaminación, medio ambiente, quitina, vertimientos.

\section{ABSTRACT}

Waste water disposed in a superficial current, lakes, rivers, the sea, without any treatment, causes great inconvenient pollution that affects the flora and fauna of a region. This wastewater, before being poured into the receiving body of water, must receive the appropriate treatment, in order to modify its physical, chemical and microbiological conditions, to avoid that its disposal causes the alteration and degradation of the associated ecosystems and problem with public health. This research had as an objective to assess the effect of chitosan and aqueous extract of orange peel shells at different concentrations on the coagulation-flocculation process of wastewater. In order to do this, a jar test with fast and slow pull was carried out, assessing turbidity (NTU), chemical oxygen demand (COD), biochemical oxygen demand (BOD), total suspended solids (TSS) and SS (solid sediments). The results showed that all the treatments presented significant 
differences $(p<0.05)$ to the control sample, and were efficient in the decrease of NTU, DQO, DBO, SST, and SS. The treatment that combined chitosan and aqueous extract of orange peel (50-50\%) at a $\mathrm{pH}$ or 5.5, decreased significantly $(p<0.05)$ the turbidity in a $79 \%$, showing in a preliminary way that the aqueous extract of orange peel shells acidifies the mix and increases the formation of agglomerated floccules in a light phase of the samples, becoming an efficient agent to be used in the treatment of wastewater.

Keywords: organic load, coagulants, pollution, environment, chitin, discharges.

\section{INTRODUCCIÓN}

La escasez de agua potable es un fenómeno inducido por los seres humanos, al ser desperdiciada, contaminada y manejada de manera insostenible, convirtiéndose en un grave problema de salud pública, en muchos países del mundo (Connor et al. 2017). El control de la contaminación del agua, se centra en la identificación, la clasificación y la prioridad de los problemas locales, relacionados con la calidad del agua y el control de la contaminación, para la protección de la salud y del medio ambiente (Tchounwou et al. 2012). Una de las principales fuentes de contaminación de las aguas superficiales son los vertimientos de agua residual doméstica e industriales, provenientes de los sistemas de alcantarillados municipales (Nkansah et al. 2016). Los tratamientos de agua residuales buscan la reducción de la carga orgánica contaminante (Connor et al. 2017).

Estas aguas tratadas, posteriormente, son incorporadas a cuerpos de aguas naturales, como ríos, quebradas, caños, entre otros, siendo nuevamente fuente de captación para uso agrícola, pecuario y a plantas de tratamiento de agua potable municipales, para disminuir la carga de microorganismos patógenos en estas (Thebe \& Mangore, 2012). Las técnicas convencionales para tratamiento de aguas residuales presentan dificultades al no cumplir con los límites de vertido, ocasionando efectos adversos sobre los organismos presentes en el ecosistema acuático, haciendo necesario la implementación de procesos alternos de depuración de aguas residuales, como lo es la utilización de material orgánico, implementando una tecnología limpia y amigable con el medio ambiente (Clemente et al. 2013).

Para el tratamiento de las aguas residuales, los tratamientos más utilizados son procesos físico-químicos, tales como la electrocoagulación, ozonización, fotocatálisis, filtración por membrana, biodegradación, coagulación-floculación y adsorción (Barrios et al. 2015). Asimismo, se han empleado diversos agentes coagulantes, como sales metálicas hidrolizables, cloruro férrico, sulfato de aluminio, policloruro de aluminio y polímeros biodegradables con cargas definidas, a través de su estructura, para reducir los niveles de contaminación.

El quitosano es un polisacárido lineal, que se puede obtener de diversos residuos, hongos, caparazones de crustáceos e insectos y muestra actividad antimicrobiana contra diferentes hongos, bacterias Gram positivas y negativas (Verlee et al. 2017; Zou et al. 2016).

El quitosano es un copolímero al azar de D-glucosamina y $\mathrm{N}$-acetil-D unidades de glucosamina. La relación entre estas dos unidades monoméricas, expresada como el grado de acetilación o desacetilación y el peso molecular, juegan un papel importante en la actividad antimicrobiana del quitosano y la solubilidad en medios acuosos (Verlee et al. 2017).

El quitosano se ha descrito como un polímero catiónico lineal, biodegradable, no tóxico, de alto peso molecular de fácil aplicación y ambientalmente amigable. Se ha demostrado que la presencia de los grupos aminos en la estructura del quitosano confiere la capacidad para coagular sustancias coloidales; además, su uso permite aumentar la acción de coagulantes inorgánicos convencionales (Caldera et al. 2009).

En la última década, la cáscara de naranja ha sido utilizada para obtener varios productos de valor agregado, tales como: aceites esenciales, pectina, antioxidantes naturales, antimicrobiano, etanol, ácidos orgánicos y oligosacáridos pectóticos (Mamma \& Christakopoulos, 2014). La cáscara de naranja está compuesta principalmente de celulosa, pectina, hemi-celulosa, lignina, pigmentos de clorofila y otros hidrocarburos de bajo peso molecular, que contienen muchos grupos funcionales hidroxilo, lo que hace que sea un adsorbente potencial para muchos contaminantes (Bhatnagar et al. 2015). Posee componentes bioactivos, que se considera que tienen un efecto beneficioso sobre la salud, especialmente, en compuestos fenólicos y polifenólicos, que exhiben actividades antimicrobianas y antioxidantes (Ahn et al. 2007; Benites et al. 2011).

El objetivo de esta investigación fue realizar una evaluación preliminar del efecto del quitosano, del extracto acuoso de la cáscara de naranja y su combinación en la coagulación-floculación de aguas residuales.

\section{MATERIALES Y MÉTODOS}

Localización. La investigación, se llevó a cabo durante junio de 2017, en el Laboratorio de la planta de tratamiento de aguas residuales de Empresa Comunitaria de Acueducto, Alcantarillado y Aseo Saravena, Arauca, Colombia. La temperatura media diurna dentro del laboratorio fue de $27 \pm 2{ }^{\circ} \mathrm{C}$ y una humedad relativa del $74 \%$. 
Caracterización del agua contaminada. Las muestras, se tomaron en el punto de vertimiento final del sistema de tratamiento de agua residual del municipio de Saravena, en Arauca-Colombia, de acuerdo al instructivo del IDEAM. Los análisis, se efectuaron en el laboratorio acreditado SIAMA LTDA. $N^{\circ}$ : P171164 de Bucaramanga, se realizaron las pruebas de: turbidez, dureza, $\mathrm{pH}$, demanda química de oxígeno (DQO), demanda bioquímica de oxígeno (DBO), sólidos suspendidos totales (SST), sólidos sedimentables (SS).

Preparación de soluciones de quitosano. El quitosano, comercialmente disponible, (Ch) de bajo peso molecular, con un grado de desacetilación (DA) del 81,4\% (50.000-190.000 $\mathrm{Da})$, se adquirió de la empresa ALDRICH. El polvo de quitosano $(1 \mathrm{~g})$, se mezcló con $5 \mathrm{~mL}$ de solución de ácido acético, al $1 \%$, aforando a $100 \mathrm{~mL}$ con agua destilada, con agitación constante por $1 \mathrm{~h}$, a temperatura ambiente, para obtener una solución de quitosano al 1\% (p/v) (Álava, 2015; Paz et al. 2012).

Obtención del extracto acuoso de cáscara de naranja. Se pesaron $2 \mathrm{~g}$ de cáscara de naranja molidas y se agregó $200 \mathrm{~mL}$ de agua, a temperatura de ebullición, dejándola reposar por 3 min. Se filtró en caliente, obteniéndose soluciones al $1 \%$, que se almacenaron a temperatura de 0 a $4^{\circ} \mathrm{C}$, en frascos ámbar, hasta su uso (Domínguez, 2016).
Proceso de coagulación-floculación. Se utilizó un equipo de Prueba de Jarra modelo JLT4; se agregó 2L de la muestra de agua residual, a cada uno de los cuatro vasos de precipitado, tomando uno de estos como control. Posteriormente, se procedió a agregar $1 ; 1,5$ y $2 \%$ de quitosano y extracto acuoso de la cáscara de naranja; las mezclas, se agitaron en dos etapas rápida, a $250 \mathrm{rpm}$, por $5 \mathrm{~min}$ y lenta, a 40rpm, por $20 \mathrm{~min}$, a $25 \pm 2^{\circ} \mathrm{C}$ y pH de 5,5 (para ajustar el $\mathrm{pH}$, se utilizó ácido sulfúrico al $0.1 \mathrm{~N}$ ); los ensayos se realizaron por triplicado (Álava, 2015). Para la caracterización de las muestras de aguas residuales, se realizaron en SIAMA LTDA., acreditadas por IDEAM Resolución 0833/2016, Ext. Resolución 0805/2017, donde se determinó la turbidez en un turbidímetro marca HACH, dureza (método SM 2340C), pH (método SM2310B), demanda química de oxígeno DQO (mg/l)(método SM5220C), demanda bioquímica de oxígeno (DBO) (método SM5210B), sólidos suspendidos totales (SST)(método SM2540D), sólidos sedimentables (SS) (método SM2540F); se midieron antes y después del tratamiento.

Diseño experimental. Se planteó un diseño experimental completo al azar, de tres niveles (Tabla 1), con el objeto de evaluar el efecto que tienen el quitosano y el extracto acuoso de cáscara de naranja en el tratamiento de aguas residuales. Este proceso, se realizó en la prueba de jarras, a pH de 5,5, teniendo como variable de respuesta al porcentaje de remoción de turbiedad, para un volumen de muestra de $2 \mathrm{~L}$.

Tabla 1. Factores evaluados para el tratamiento de las muestras de aguas residuales.

\begin{tabular}{|c|c|c|c|c|c|}
\hline \multirow{2}{*}{ Factores } & \multicolumn{3}{|c|}{ Niveles Concentración } & \multirow{2}{*}{ Réplicas } & \multirow{2}{*}{$\begin{array}{l}\text { Variable de } \\
\text { respuesta }\end{array}$} \\
\hline & \multicolumn{3}{|c|}{ (\%) } & & \\
\hline Quitosano(Q) & 1 & 1,5 & 2 & \multirow{3}{*}{3} & \multirow{3}{*}{$\begin{array}{c}\text { Porcentaje de } \\
\text { remoción } \\
\text { de turbidez \% } \\
\text { DBO } \\
\text { DQO } \\
\text { SST } \\
\text { SS }\end{array}$} \\
\hline $\begin{array}{c}\text { Extracto acuoso de cascara } \\
\text { de naranja (EACN) }\end{array}$ & 1 & 1,5 & 2 & & \\
\hline $\begin{array}{c}\text { Quitosano + extracto } \\
\text { acuoso de la cascara de } \\
\text { naranja }(Q+E A C N)\end{array}$ & $0,5+0,5$ & $0,75+0,75$ & $1+1$ & & \\
\hline
\end{tabular}

Análisis estadístico. Los resultados obtenidos fueron tratados estadísticamente significativa, a un nivel de 95\% $(\mathrm{P}<0,05)$; con el fin de discernir mejor los resultados del ANOVA, se utilizó la prueba post hoc de Diferencias Mínimas Significativas (DMS), paquete de software estadístico SPSS versión 22.0.

\section{RESULTADOS Y DISCUSIÓN}

En los resultados, se puede observar que todas las muestras superan el valor máximo permisible de $200 \mathrm{mg} \mathrm{O} / \mathrm{L}$ del parámetro de $\mathrm{DQO}$ de la Resolución 0631/2015, que establece los valores límites permisibles en los vertimientos puntuales, a cuerpos de agua superficiales. 
La figura 1 muestra que todos los tratamientos fueron eficientes en la remoción de materia orgánica (DQO), presentando diferencias significativas $(p<0,05)$ con la muestra control. El tratamiento que combina quitosano + extracto acuso de cáscara de naranja en sus tres concentraciones (1;1,5 y $2 \%)$, obtuvieron el mayor porcentaje de remoción (45\%), indicando la efectividad para retirar la materia orgánica de las muestras de aguas residuales.
Caldera et al. (2011) reportaron remociones de DQO en las aguas de la industria petrolera, no superiores al $60 \%$, al ser tratadas con quitosano, mientras que Caldera et al. (2015) observaron una remoción eficiente en la de materia orgánica (DQO), al tratar con quitosano aguas de la industria avícola, con remociones entre 71 y $81 \%$.

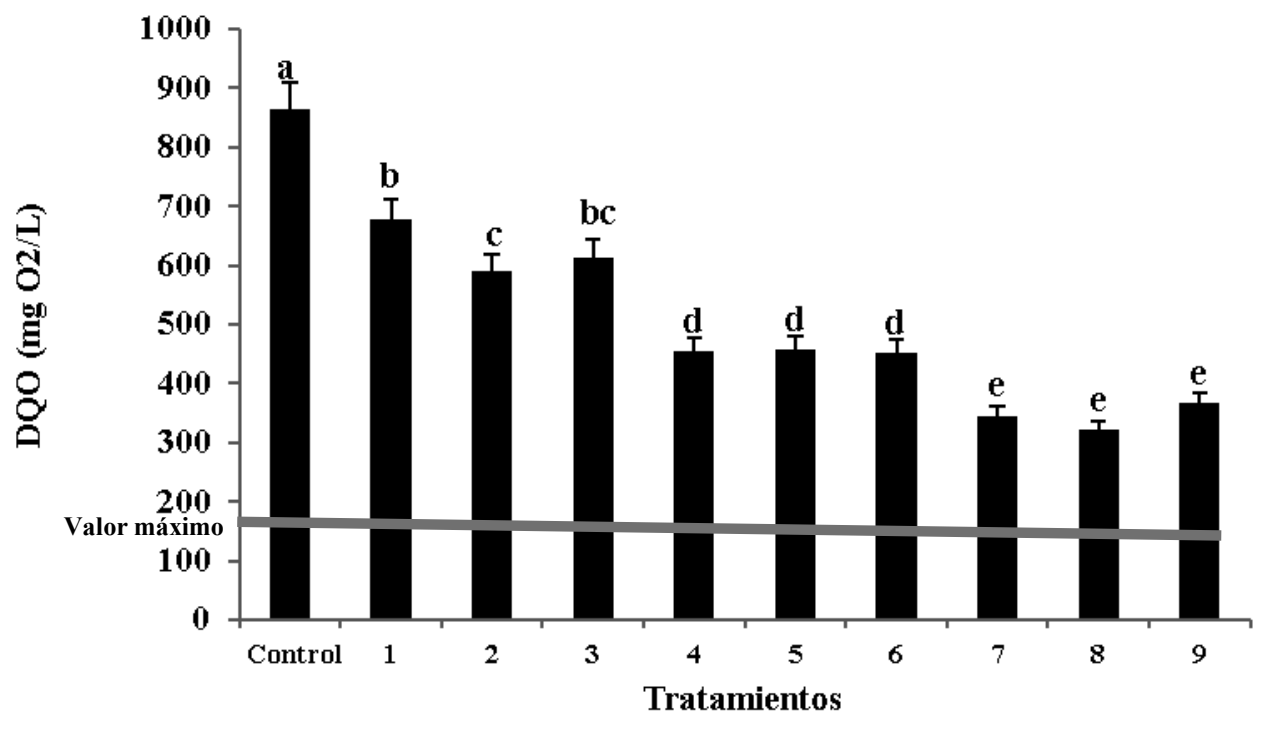

Figura 1. Resultados del parámetro de $\mathrm{DQO}$ de las muestras de aguas residuales tratadas con quitosano y extracto acuso de cáscara de naranja.

El pH es uno de los factores de mayor importancia y efecto sobre el proceso de coagulación; su rango óptimo para la remoción de coloides depende de la naturaleza del agua y puede variar entre 5,0 y 8,0 unidades (Romero, 2005). Las muestras de agua residuales presentaron un $\mathrm{pH}$ de 7,21 neutro. Estudios realizados por Selmer et al. (1996), concluyeron que el quitosano funciona, efectivamente, como coagulante a rangos de $\mathrm{pH}$ superiores a 5,25 unidades (Caldera et al. 2009; Caldera et al. 2011).

La figura 2 muestra que todos los tratamientos fueron eficaces en la disminución de los valores del parámetro de DBO, presentando diferencias significativas $(p<0,05)$ con la muestra control. El tratamiento de quitosano y la mezcla de quitosano con extracto acuoso de cáscara de naranja en sus tres concentraciones, obtuvieron las mayores remociones de un 27 y $40 \%$, respectivamente.

En los resultados, se puede observar que los valores de DBO (Figura 2) son menores en comparación con DQO, derivan- do una relación de la fracción $\mathrm{DBO} / \mathrm{DQO}$ baja $(0,48)$, lo que indica que los tratamientos biológicos no son la mejor opción, puesto que se ha reportado que esta relación debería ser superior a 0,5, para la fácil degradación por los microorganismos (Tchobanoglous \& Crites, 2003; Caldera et al. 2011). Esto justifica la alternativa del proceso fisicoquímico para remover los contaminantes presentes en las en aguas residuales (Tondwal \& Singh, 2017).

En la tabla 2, se presentan los valores de turbidez después de efectuar los tratamientos de agua residual con quitosano comercial y extracto acuso de cáscara de naranja, para las diferentes concentraciones evaluadas $(1,0 ; 1,5$ y $2,0 \%)$. La muestra control presentó diferencias significativas $(p<0,05)$ con los demás tratamientos; la mezcla de quitosano y extracto acuoso de la cáscara de naranja en sus tres concentraciones, mostraron una disminución de 173 a 36 NTU, con una remoción de un $79 \%$. 


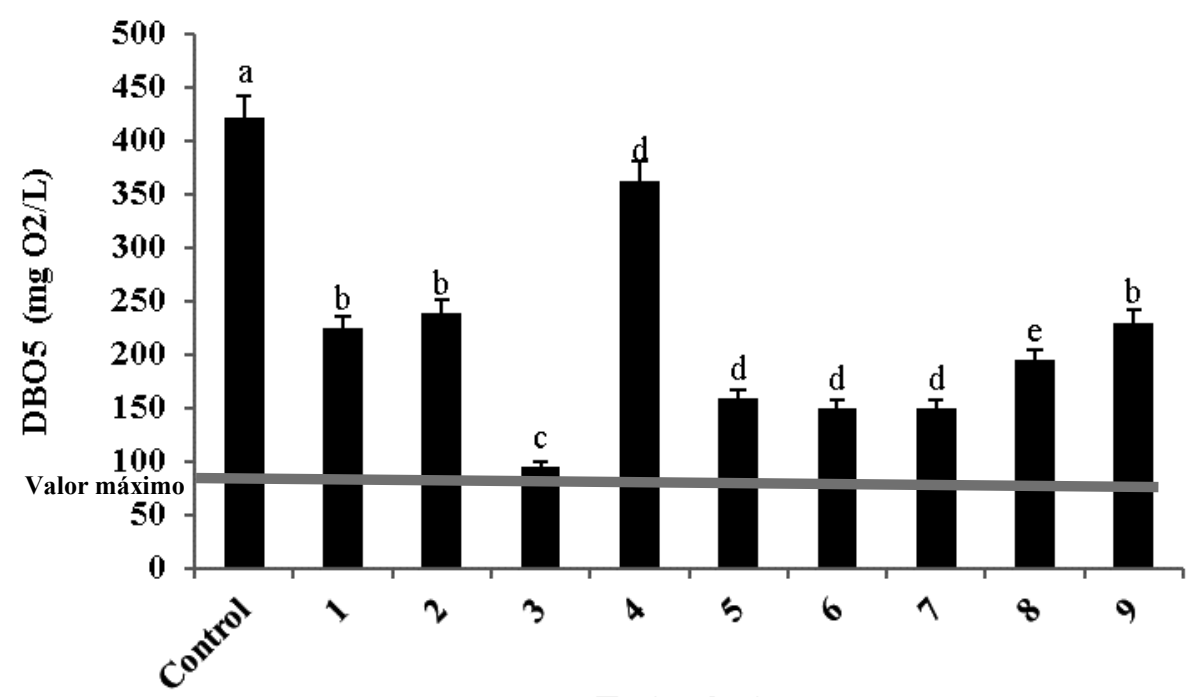

Tratamiento

Figura 2. Resultados del parámetro de DBO de las muestras de aguas residuales tratadas con quitosano y extracto acuoso de la cáscara de naranja.

Con respecto a la concentración de SS y SST de las muestras de aguas residuales, se observa que no se cumplen con los valores límites permisibles establecidos en la Resolución 0631/2015. Los tratamientos logran reducir los valo- res de estos parámetros mostrando diferencias significativas $(p<0,05)$ con la muestra control. El tratamiento que combina el quitosano con extracto acuoso de la cáscara de naranja en sus tres concentraciones presentó una disminución

Tabla 2. Parámetros evaluados de los tratamientos de las aguas residuales con quitosano comercial y extracto acuoso de la cáscara de naranja.

\begin{tabular}{|c|c|c|c|c|}
\hline \multirow{2}{*}{ Parámetro } & Turbidez & Dureza total & $\begin{array}{c}\text { Sólido } \\
\text { Sedimentable }\end{array}$ & $\begin{array}{c}\text { Sólido Suspendidos } \\
\text { Totales }\end{array}$ \\
\cline { 2 - 5 } & $\mathbf{( N T U )}$ & $(\mu \mathrm{m} \mathrm{CaCO} / \mathrm{L})$ & $(\mu \mathrm{m} / \mathrm{L})$ & $\mathbf{\mu m} / \mathrm{L})$ \\
\hline Método & - & AM2340C & SM2540F & SM2540D \\
\hline Resolución 0631/2015 & No reporta & Análisis y reporte & 5,0 & 100,00 \\
\hline Control & $173 \pm 13^{\mathrm{a}}$ & $31 \pm 3^{\mathrm{a}}$ & $20 \pm 2^{\mathrm{a}}$ & $1021 \pm 132^{\mathrm{a}}$ \\
\hline $1(\mathrm{Q}-1 \%)$ & $42 \pm 2^{\mathrm{b}}$ & $39 \pm 5^{\mathrm{b}}$ & $13 \pm 4^{\mathrm{b}}$ & $590 \pm 65^{\mathrm{b}}$ \\
\hline $2(\mathrm{Q}-1,5 \%)$ & $45 \pm 3^{\mathrm{b}}$ & $49 \pm 3^{\mathrm{c}}$ & $9 \pm 4^{\mathrm{b}}$ & $514 \pm 38^{\mathrm{b}}$ \\
\hline $3(\mathrm{Q}-2 \%)$ & $44 \pm 2^{\mathrm{b}}$ & $39 \pm 4^{\mathrm{b}}$ & $10 \pm 2^{\mathrm{b}}$ & $611 \pm 74^{\mathrm{b}}$ \\
\hline $4(\mathrm{EN}-1 \%)$ & $72 \pm 4^{\mathrm{c}}$ & $74 \pm 4^{\mathrm{d}}$ & $14 \pm 3^{\mathrm{b}}$ & $621 \pm 82^{\mathrm{b}}$ \\
\hline $5(\mathrm{EN}-1,5 \%)$ & $66 \pm 2^{\mathrm{c}}$ & $75 \pm 2^{\mathrm{d}}$ & $11 \pm 4^{\mathrm{b}}$ & $644 \pm 56^{\mathrm{b}}$ \\
\hline $6(\mathrm{EN}-2 \%)$ & $67 \pm 3^{\mathrm{c}}$ & $79 \pm 3^{\mathrm{d}}$ & $14 \pm 2^{\mathrm{b}}$ & $626 \pm 83^{\mathrm{b}}$ \\
\hline $7(\mathrm{Q}+\mathrm{EN} 1 \%)$ & $39 \pm 1^{\mathrm{d}}$ & $53 \pm 4^{\mathrm{e}}$ & $10 \pm 2^{\mathrm{b}}$ & $451 \pm 24^{\mathrm{c}}$ \\
\hline $8(\mathrm{Q}+\mathrm{EN} 1,5 \%)$ & $36 \pm 2^{\mathrm{d}}$ & $63 \pm 3^{\mathrm{f}}$ & $12 \pm 3^{\mathrm{b}}$ & $444 \pm 27^{\mathrm{c}}$ \\
\hline $9(\mathrm{Q}+$ EN 2\%) & $36 \pm 3^{\mathrm{d}}$ & $55 \pm 4^{\mathrm{e}}$ & $11 \pm 1^{\mathrm{b}}$ & $418 \pm 38^{\mathrm{c}}$ \\
\hline
\end{tabular}

* \pm Desviación estándar-Diferentes letras en la misma columna indican diferencias mínimas significativas DMS (p <0,05).

Q: Quitosano, EN: Extracto acuoso de cáscara de naranja, Q+EN: Quitosano + acuoso de la cáscara de naranja. 
del $42 \%$ en los valores de los sólidos suspendidos totales, debido, probablemente, a la sedimentación de los lodos biológicos, en la etapa de clarificación. Los tratamientos no presentaron diferencias significativas $(p<0.05)$ en los valores reportados del parámetro de sólidos sedimentables.

Durante la prueba de jarras, se observó que al incrementar la dosis de concentración de los tratamientos, el tamaño de los flóculos era mayor, con una rápida velocidad de sedimentación, debido, posiblemente, al mecanismo de coagulación que presenta el quitosano, al poseer una cadena larga de grupos amino e hidroxilo, ubicados en su estructura, interaccionando con las partículas coloidales (Zhang et al. 2018). Esto le permite actuar al quitosano como un puente entre las partículas, que adsorben los iones con carga opuesta del quitosano, neutralizando las cargas y logrando, de esta manera, la desestabilización de los coloides en las muestras de aguas residuales (Zhang et al. 2018; Balanta et al. 2010; Mármol et al. 2004).

El quitosano actúa como agente coagulante en aguas residuales, por ser polímero catiónico, efecto relacionado a la desestabilización de los coloides y contribuyen a la clarificación; el grupo amino puede proporcionar carga positiva y unirse con metales (Zhang et al. 2018; Martínez et al. 2018). Rojas et al. (2008) señalan remociones de turbidez en guas de producción de petróleo de más del $90 \%$, durante el tratamiento con polímeros catiónicos, mientras que Selmer et al. (1996), refieren $90 \%$ de remoción de la turbidez presente en aguas residuales de industrias lácteas después del tratamiento con quitosano (Caldera et al. 2009; Caldera et al. 2011).

Resultados similares obtuvieron Caldera et al. (2015), en el parámetro de turbidez, al obtener remociones superiores al $76 \%$, después de tratar con quitosano aguas residuales industriales y las aguas sintéticas con alta turbidez. Estudios indican que el quitosano es un biomaterial con excelentes características coagulantes para procesos de tratamiento de las aguas residuales de alta turbidez y alcalinidad, debido a su tamaño molecular y alta viscosidad, que puede conducir a una mejor coagulación (Zhang et al. 2018; Tondwal \& Singh, 2017).

Los tratamientos con extracto acuoso de la cáscara de naranja en sus tres concentraciones mostraron una disminución de 173 a 68 NTU, con una remoción de un 39\% y un descenso en los demás parámetros, de manera significativa ( $p<0,05)$, en comparación con la muestra control. El contenido porcentual de los componentes de la cáscara de naranja en azúcares solubles $(16,9 \%)$, celulosa $(9,21 \%)$, hemicelulosa $(10,5 \%)$ y pectina $(42,5 \%)$, han revelado poseer capacidad coagulante (Pinzón \& Cardona, 2010; Tejada et al. 2015).
Las peptinas contienen, al menos, $65 \%$ de unidades de ácido galacturónico que es, posiblemente, un ingrediente activo, que proporciona la capacidad de coagulación. Ensayos en combinación con otros azúcares, presentes en la cáscara de naranja, mostraron reducciones de turbidez del 50\% (Miller et al. 2008; Voragen et al. 2003; Contreras et al. 2015).

Estos resultados, probablemente, se deban a que el ácido galacturónico predomina en especies vegetales en forma polimérica (ácido poligalacturónico), cuya estructura indica que es aniónico y la existencia de grupos carboxílicos, a lo largo de la cadena, implica que se puede dar la absorción química entre partículas cargadas suspendidas en el agua y estos grupos funcionales (Contreras et al. 2015).

Estudios recientes de Nithya \& Abirami (2018) mostraron que al utilizar $0,6 \mathrm{gm} / \mathrm{mL}$ de quitosano, a un $\mathrm{pH}$ de 6 , se logra la eliminación de la turbidez, en un 91,3\%. Los resultados muestran una posible relación entre estas propiedades fisicoquímicas, ya que la turbidez es el producto de una variedad de partículas disueltas, incluyendo los sedimentos suspendidos, de partículas inorgánicas o fuentes biológicas, siendo un sistema económico, efectivo y método seguro; para la eliminación de contaminantes en el lixiviado ha resultado en el proceso de coagulación.

Conflictos de intereses: El manuscrito fue preparado y revisado con la participación de todos los autores, quienes declaramos que no existe conflicto de intereses que ponga en riesgo la validez de los resultados presentados. Financiación: Este estudio fue financiado por Servicio Nacional de Aprendizaje-SENA.

\section{REFERENCIAS}

1. AHN, J.; KIM, Y.; SEO, E.; CHOI, Y.; KIM, H. 2007. Antioxidant effect of natural plant extraets on the microencapsulated nigh oleic sunflower oil. J. Food Engineering. 84:327-334. https://doi.org/10.1016/j. jfoodeng.2007.05.029

2. ÁLAVA, J.M. 2015. Aplicación de quitosano como biocoagulante en aguas residuales contaminadas con hidrocarburos. Enfoque UTE. 6(3):52-64. https://doi. org/10.29019/enfoqueute.v6n3.69

3. BALANTA, D.; GRANDE, C.; ZULUAGA, F. 2010. Extracción, identificación y caracterización de quitosano del micelio de Aspergillus niger y sus aplicaciones como material bioadsorbente en el tratamiento de aguas. Iberoam. Polim. 11(5):297-316. 
4. BARRIOS, L.F.; GAVIRIA, L.F.; AGUDELO, E.A.; CARDONA, S.A. 2015. Technologies for the removal of dyes and pigments present in wastewater. A review. DYNA. 82:118126. http://dx.doi.org/10.15446/dyna.v82n191.42924

5. BENITES, J.; DÍAZ, R.; LÓPEZ, J.; GAJARDO, S. 2011. Actividad antioxidante y antibacteriana de seis cáscaras de frutos del oasis de Pica. Rev. Biofarbo. 19(1):1-7.

6. BHATNAGAR, A.; SILLANPA, A.; WITEK, A. 2015. Agricultural waste peels as versatile biomass for water purification - a review. Chem. Eng. J. 270:244-271. https://doi.org/10.1016/j.cej.2015.01.135

7. CALDERA, Y.; CLAVEL, N.; BRISEÑO, D.; NAVA, A.; GUTIÉRREZ, E.; MÁRMOL, Z. 2009. Quitosano como coagulante durante el tratamiento de agua de producción de petróleo. Boletín del Centro de Investigaciones Biológicas. 43(4):541-555.

8. CALDERA, Y.; RODRÍGUEZ, Y.; OÑATE, H.; PRATO, J.; GUTIÉRREZ, E. 2011. Eficiencia del quitosano como coagulante durante el tratamiento de aguas de baja turbidez asociadas a la producción de petróleo. Rev. Tecnocientífica URU. 1(1):45-52.

9. CALDERA, Y.; GUTIÉRREZ, E.; FUENTES, L.; MENDOZA, I.; RODRÍGUEZ, Y.; SÁNCHEZ, M. 2015. Quitosano: un coagulante natural alternativo para el tratamiento de aguas con alta turbidez. REDIELUZ. 5(1-2):184188.

10. CLEMENTE, A.R.; ARRIETA, E.L.; MESA, G.A. 2013. Procesos de tratamiento de aguas residuales para la eliminación de contaminantes orgánicos emergentes. Rev. Ambiente \& Água. 8(3):93-103. http:// dx.doi.org/10.4136/ambi-agua. 1176

11. CONNOR, R.; RENATA, A.; ORTIGARA, C.; KONCAGÜL, E.; UHLENBROOK, S.; LAMIZANA-DIALLO, B.M.; HENDRY, S. 2017. The United Nations World Water Development Report 2017. Wastewater: The Untapped Resource. The United Nations World Water Development Report. p.16-26.

12. CONTRERAS, K.; AGUAS, Y.; SALCEDO, J.; OLIVERO, R.; MENDOZA, P. 2015. El Nopal (Opuntia ficus-indica) como coagulante natural complementario en la clarificación de agua. Producción + Limpia. 10(1):4050.

13. DOMÍNGUEZ, T. 2016. Flavonoides extraídos de la cascara de naranja tangelo (Citrus reticulata $x$ Citrus paradisi) y su aplicación como antioxidante natural en el aceite vegetal sacha inchi (Plukenetia volubilis). Scientia Agropecuaria. 7(4):419-431. http://dx.doi. org/10.17268/sci.agropecu.2016.04.07

14. MAMMA, D.; CHRISTAKOPOULOS, P. 2014. Biotransformation of citrus by-products into value added products. Waste and Biomass Valorization. 5:529-549. https://doi.org/10.1007/s12649-013-9250-y

15. MÁRMOL, Z.; GUTIÉRREZ, E.; PÁEZ, G.; FERRER, P.; RINCÓN, M. 2004. Desacetilación termoalcalina de quitina de conchas de camarón. Multiciencias. 4:9195.

16. MARTÍNEZ, M.; LÓPEZ, E.; OCHOA, A.; PINA, G.; OROPEZ, M. 2018. Modification of chitosan with carbamoyl benzoic acids for testing its coagulantflocculant and binding capacities in removal of metallic ions typically contained in plating wastewater. Chemical Engineering J. 332:749-756. https://doi. org/10.1016/j.cej.2017.09.042

17. MILLER, S.; FUGATE, E.; CRAVER, V.; SMITH, J.; ZIMMERMAN, J. 2008. Toward understanding the efficacy and mechanisim of Opuntia spp. as a natural coagulant for potential application in water treatment. Environmental Science and Technology. 42(12):42744279. http://doi.org/10.1021/es7025054

18. MINISTERIO DE MEDIO AMBIENTE Y DESARROLLO SOSTENIBLE, MINAMBIENTE. 2015. Resolución 0631 de 2015. Parámetros y los valores límites máximos permisibles en los vertimientos puntuales a cuerpos de aguas superficiales, Bogotá, Colombia.

19. NITHYA, M.; ABIRAMI, M. 2018. The leachate treatment by using natural coagulants (pine bark and chitosan). Internal Res. J. Engineering and Technology (IRJET). 5(4):2711-2714.

20. NKANSAH, M.A.; OPOKU, F.; EPHRAIM, J.H.; WEMEGAH, D.D.; TETTEH, L.P. 2016. Characterization of beauty salon wastewater from Kwame Nkrumah University of Science and Technology, Kumasi, Ghana, and its surrounding communities. Environmental Health Insights.10:147-154. https://doi.org/10.4137/ EHI.S40360

21. PAZ, N.D.; FERNÁNDEZ, M.; LÓPEZ, O.D.; NOGUEIRA, A.; GARCÍA, C.M.; PÉREZ, D.; DÍAZ, D. 2012. Optimización del proceso de obtención de quitosano derivada de la quitina de langosta. Rev. Iberoam. Polímeros. 13(3):103-116. 
22. PINZÓN-BEDOYA, M.L.; CARDONA TAMAYO, A.M. 2010. Influencia del pH en la bioadsorción de $\mathrm{Cr}$ (III) sobre cáscara de naranja: Determinación de las condiciones de operación en proceso discontinuo. Rev. Fac. Ciencias Básicas. 8(1):21-30.

23. ROJAS, C.; RINCÓN, N.; DÍAZ, A.; COLINA, G.; BEHLLNG, E.; CHACÍN, E.; FERNÁNDEZ, N. 2008. Evaluación de una unidad de flotación con aire disuelto para el tratamiento de aguas aceitosas. Rev. Téc. Ing. Univ. Zulia. 3:50-57.

24. ROMERO, A. 2005. Calidad del agua ( $2^{\mathrm{a}}$ ed.). Editorial Escuela Colombiana de Ingeniería, Bogotá, Colombia. 468.p.

25. SELMER, E.; RATNAWEERA C.; PEHRSON, Y.R. 1996. A novel treatment process for dairy wastewater with chitosan produced from shrimp-shell waste. Wat. Sci. Tech. 34:33-40. https://doi.org/10.1016/S02731223(96)00818-9

26. TCHOBANOGLOUS, G.; CRITES, R. 2003. Tratamiento de Aguas Residuales en pequeñas poblaciones. Bogotá, Colombia: Mc Graw Hill. 776p.

27. TCHOUNWOU, P.; YEDJOU, C.; PATLOLLA, A.; SUTTON, D.J. 2012. Heavy metal toxicity and the environment. In Molecular, clinical and environmental toxicology. Experientia Supplementum. 101:133-164. https://doi.org/10.1007/978-3-7643-8340-4_6

28. TEJADA, C.; VILLABONA, A.; GARCÉS, L. 2015. Adsorción de metales pesados en aguas residuales usando materiales de origen biológico. Tecno Lógicas. 18(34):109-123.
29. THEBE, T.A.; MANGORE, E.N. 2012. Wastewater production, treatment, and use in Zimbabwe. The UN-Water Activity Information System (UNW-AIS). P.1-10.

30. TONDWAL, R.; SINGH, M. 2017. Chitosan functionalization with a series of sulfur-containing $\alpha$-amino acids for the development of drug-binding abilities. J. Applied Polymer Science. 135(12):46000. https:// doi.org/10.1002/app.46000

31. VERLEE, A.; MINCKE, S.; STEVENS, C.V. 2017. Recent Developments in Antibacterial and Antifungal Chitosan and Its Derivatives. Carbohydr. Polym. 164:268283. https://doi.org/10.1016/j.carbpol.2017.02.001

32. VORAGEN, A.; SCHOLS, H.; VISSER, R. 2003 Advances in Pectin and Pectinase Research. Kluwer Academic Publishers, Dordrecht, The Netherlands. 491p.

33. ZHANG, Z.; JING, R.; HE, S.; QIAN, J.; ZHANG, K.; MA, G.; CHANG, X.; ZHANG, M.; LI, Y. 2018. Coagulation of low temperature and low turbidity water: Adjusting basicity of polyaluminum chloride (PAC) and using chitosan as coagulant aid. Separation and Purification Technology, 206:131-139. http://dx.doi. org/10.1016/j.seppur.2018.05.051

34. ZOU, P.; YANG, X.; WANG, J.; LI, Y.; YU, H.; ZHANG, Y. 2016. Advances in haracterization and biological activities of chitosan and chitosan oligosaccharides. Food Chem. 190:1174-1181. https://doi. org/10.1016/j.foodchem.2015.06.076

Recibido: Abril 16 de 2018

Aceptado: Octubre 18 de 2018

Cómo citar:

Campo Vera, Y.; Delgado, M.A.; Roa, Y.; Mora, G.; Carreño Ortiz, J. 2018. Evaluación preliminar del efecto del quitosano y cascara de naranja en la coagulación-floculación de aguas residuales. Rev. U.D.C.A Act. \& Div. Cient. 21(2):565-572. https:// doi.org/10.31910/rudca.v21.n2.2018.990 